This document is the Accepted Manuscript version of a Published Work that appeared in final form in Langmuir, copyright 2015 (C) American Chemical Society after peer review and technical editing by the publisher. To access the final edited and published work see http://pubs.acs.org/doi/abs/10.1021/acs.langmuir.5b00306

\title{
Adsorption of surface-modified silica nanoparticles to the interface of melt poly (lactic acid) and supercritical carbon dioxide
}

\author{
K. Sarikhani ${ }^{a, b}$, K. Jeddi, ${ }^{a, b}$, R.B. Thompson ${ }^{b, c}$, C.B. Park ${ }^{d}$, P. Chen ${ }^{a, b^{*}}$ \\ ${ }^{a}$ Department of Chemical Engineering, University of Waterloo, 200 University Avenue, Waterloo, \\ Ontario, Canada N2L $3 G 1$ \\ ${ }^{b}$ Waterloo Institute for Nanotechnology, University of Waterloo, 200 University Avenue, Waterloo, \\ Ontario, Canada N2L $3 G 1$ \\ ${ }^{c}$ Department of Physics and Astronomy, University of Waterloo, 200 University Avenue, Waterloo, \\ Ontario, Canada N2L $3 G 1$ \\ ${ }^{d}$ Microcellular Plastics Manufacturing Laboratory, Department of Mechanical and Industrial \\ Engineering, University of Toronto,5 King's College Road, Toronto, Ontario, Canada M5S $3 G 8$
}

\section{Abstract}

With the purpose of fabricating polymer nanocomposite foams and preventing coalescence in foaming processes, the interfacial tension of poly (lactic acid) (PLA) - silica composites is investigated in this work. Synthesized silica nanoparticles (SNs) with a $\mathrm{CO}_{2}$ - philic surface modification are used as the dispersed nanoparticles. Interfacial tension is a key parameter in processing of polymer foams since it directly affects the final foam properties, such as cell size and cell density. Interfacial tension of silica-containing PLA and supercritical carbon dioxide $\left(\mathrm{CO}_{2}\right)$ is measured using Axisymmetric Drop Shape Analysis Profile (ADSA-P) pendant drop method at high pressures and high temperatures. The interfacial tension between PLA and supercritical $\mathrm{CO}_{2}$ is observed to decrease as a result of nanoparticles' adsorption to the interface. These results indicate that the reduction in interfacial tension with increasing silica content significantly deviates from a linear trend; there is a minimum at $2 \mathrm{wt}$. \% loading of the SNs and then the interfacial tension curve reaches a plateau. Contact angle measurements show an affinity of the SNs for the polymer-supercritical $\mathrm{CO}_{2}$ interface, and these obtained results are used to calculate the binding energy of the nanoparticles to the PLA / $\mathrm{CO}_{2}$ interface. In addition to interfacial properties, the adsorption of silica nanoparticles at the interface is also studied in detail with Scanning Electron Microscopy.

\footnotetext{
${ }^{*}$ Corresponding author. Tel.: 519-888-4567 x35586

E-mail address: p4chen@uwaterloo.ca (P. Chen).
} 


\section{Introduction}

Polymer nanocomposites are interesting materials with broad applications and exceptional mechanical, thermal, electrical, and electrochemical properties. ${ }^{1-5}$ In most cases dispersion of the nanoparticles within the polymer matrix of the nanocomposites is desired, however for some applications, such as colloidosomes, nanoparticle-armed polymer latex, Janus structures, and foams and emulsions stabilized by particles, localization is necessary. ${ }^{6-10}$

Polymeric foams have the advantages of good mechanical, energy-absorbing, and thermal-insulation properties. ${ }^{11}$ One interesting polymer for foam applications is poly (lactic acid) (PLA). This biodegradable and biocompatible polymer possesses high modulus, high strength and appropriate clarity, making it a potential replacement for petroleum-based synthetic polymers such as polypropylene, polyethylene, and poly (ethylene terephthalate). ${ }^{12}$ Furthermore, PLA has some environmental merits compared with other commodity polymers, including its renewable agricultural source, the consumption of carbon dioxide during its production, its biodegradability and recyclability. ${ }^{13,14}$

In polymeric foams made with dissolved supercritical fluids, the cell size and the cell density can be controlled by reducing the interfacial tension between the polymer and the dispersed phase (supercritical fluid). According to classical nucleation theory (CNT), the nucleation rate is inversely related to the exponential cubic power of interfacial tension; ${ }^{15,16}$ therefore one can increase the number of nucleating sites by lowering the surface tension. The decrease in surface tension decreases the energy barrier for cell nucleation and consequently increases the number of cells, leading to an exponential increase in cell densities. Moreover, lowering the surface tension results in a smaller cell size, since the critical cell size is directly related to surface tension. ${ }^{17}$ It is noteworthy that CNT with its surface tension predictions is expected to break down for nano-cellular foams, although this is beyond the scope of this paper. ${ }^{18,19}$

Similar to the role of surfactants in reducing the surface tension of foams, particles can be used to promote foam formation and stabilization. As a general concept, solid nanoparticles, such as silica, can be adsorbed at the interface to decrease the interfacial tension between polymer melts and supercritical fluids. 
Furthermore, nanoparticles can act as nucleating agents for the foaming of

polymers by increasing local stress variations around the particles (directly) ${ }^{20,21}$, and/or by promoting the crystallization of the polymers ${ }^{22}$, since the crystals can create local stress variations as the nanoparticles do (indirectly). ${ }^{23,24}$

A large number of recent studies have focused on the incorporation of solid nanoparticles as surfactants in the stabilization of foams and emulsions, provided that they are adsorbed to the fluid-gas or fluid-fluid interface, respectively, and it has been shown that the contact angle of the particles dictates the stability. ${ }^{25,26}$ For small particles, for which the effect of gravity is negligible, the energy (E) required to remove the particle from the interface is called the binding energy or adsorption energy (the free energy change upon particle adsorption to the interface), and is given by 27

$$
E=-\pi r^{2} \gamma_{\alpha \beta}(1 \pm \cos \theta)^{2}
$$

where $r$ is the radius of the particle, $\gamma_{\alpha \beta}$ is the interfacial tension between two phases (polymer-supercritical fluid in our case), and $\theta$ is the Young contact angle between the particle and the two phases (particle at the interface). The Young contact angle is defined as

$$
\cos \theta=\left(\gamma_{\alpha p}-\gamma_{\beta p}\right) / \gamma_{\alpha \beta}
$$

where $\gamma_{\alpha p}$ and $\gamma_{\beta p}$ are the interfacial tensions of the particle-polymer and particlesupercritical fluid interfaces, respectively. In the binding energy equation, considering the polymer-supercritical fluid interface with colloidal nanoparticles in the polymer phase, the sign inside the bracket is negative for removal into the polymer phase, and positive for removal into the supercritical fluid phase ,or simply the $( \pm)$ signs correlate to the cases where the particle center is above (positive) or below (negative) the interfacial plane. The particles will attach to the interface and the adsorption is irreversible if $\mathrm{E}>>\mathrm{k}_{\mathrm{B}} \mathrm{T}$, where $\mathrm{k}_{\mathrm{B}}$ is the Boltzmann constant and $\mathrm{T}$ is temperature in Kelvin. ${ }^{27-29}$

Although adsorption of solid particles to an interface can decrease interfacial tension, some self-assembled structures at the interface can increase the tension at the interface due to an increase in lateral capillary forces. The interface of the polymer melt can be deformed due to the adsorption of particles at the interface. In 
general, deformation of a liquid surface can cause a lateral capillary force. ${ }^{30}$ Depending on the particles' weight and wetting properties, the force is considered as an immersion force or a floatation force. ${ }^{31,32}$ If the force from the weight of the particles is significant and the particles are floating at the interface, the attractive or repulsive force is called a floatation force. On the other hand, for small particles partially immersed in both phases, the force is called an immersion force. The deformation of the liquid surface and magnitude of the immersion capillary force depend on the wetting properties of the particle, the magnitude of the contact angle, and the position of the contact line, and they are independent of particle weight. Equation 3 shows the amount of the capillary interaction energy between two immersed particles: ${ }^{31,33,34}$

$$
\Delta w=2 \pi \gamma Q^{2} K_{0}(q L)
$$

where $\gamma$ is the interfacial tension, $Q$ is the capillary charge of the particle, defined as $Q=r \sin \theta, \theta$ is the contact angle between a particle and the liquid at the interface, $r$ is the radius of the contact line, $q$ is defined as $q^{2}=\frac{\Delta \rho g}{\gamma}, g$ is gravity, $\Delta \rho$ is the density difference, $K_{0}$ is the modified Bessel's function of zeroth order, and $L$ is the distance between two particles. Equation 3 is valid when the distance between the particles is much smaller than the capillary length $\left(\mathrm{L} \ll q^{-1}\right)$ and also when the radii of the two contact lines is much smaller that the particle separation. It can be seen from equation 3 that for fairly close particles, the capillary interaction (even for nanoparticles) is significant.

In this work, interfacial behavior of PLA-silica nanocomposites in $\mathrm{CO}_{2}$ environment is investigated in detail. Interestingly, a non-linear trend in interfacial tension values with increasing amount of nanoparticles is observed. It is described that lateral capillary forces of the adsorbed aggregates of the nanoparticles to the PLA- $\mathrm{CO}_{2}$ interface is the reason for the reported increase in interfacial tension at higher contents of the nanoparticles.

\section{Experimental}

\subsection{Materials}

Polylactic acid (PLA), grade 2002D, with $\mathrm{M}_{\mathrm{n}}=100 \mathrm{~kg} / \mathrm{mol}$ and D-content of $4.5 \%$ was kindly provided by NatureWorks Inc. Carbon dioxide chromatographic 
grade with purity of $99.99 \%$ was purchased from PRAXAIR, Canada. Tetraethyl orthosilicate (TEOS), ammonium hydroxide (28-30\% aqueous solution), (3Aminopropyl) triethoxysilane (APTES) 99\% were purchased from Sigma-Aldrich. Deionized water (18.2 M $\Omega$ ) was obtained from a Millipore Milli-Q system.

\subsection{Synthesis of silica nanoparticles}

Silica nanoparticles were made using the well-known Stöber method. ${ }^{35}$ A typical procedure to make an $80 \mathrm{~nm}$ diameter silica particle was as follows: at room temperature, $8 \mathrm{cc}$ of tetraethylorthosilicate (TEOS; Sigma-Aldrich) was added to $100 \mathrm{cc}$ of ethanol. The $\mathrm{pH}$ was adjusted using $16 \mathrm{cc}$ of ammonium hydroxide solution $(28 \mathrm{wt} \%)$. The sol-gel reaction was carried out for four hours. Particle size was monitored using Dynamic Light Scattering (DLS), Scanning Electron Microscopy (SEM), and Transmission Electron Microscopy (TEM). After four hours, the silica was separated by centrifuging at $14000 \mathrm{rpm}$ and washed with ethanol six times. Afterwards, the silica nanoparticles were dried in an oven for 48 hours at $80^{\circ} \mathrm{C}$.

\subsection{Surface modification of silica nanoparticles}

Surface modification of silica nanoparticles was carried out through another sol-gel reaction on the surface of re-dispersed silica nanoparticles in toluene. The reaction happened between (3-aminopropyl) trimethoxysilane (APTES) and the hydroxyl functional groups on the surface of the silica nanoparticles ${ }^{36,37}$. In order to modify the surface of silica with amine functional groups, $1.6 \mathrm{~g}$ of silica nanoparticles were dispersed in $100 \mathrm{ml}$ ethanol containing $5 \mathrm{ml}$ ammonium hydroxide solution (28\%), then $3 \mathrm{ml}$ of APTES was added to the solution and stirred for $24 \mathrm{~h}$ at $75^{\circ}$ C. At the end of the reaction, the particles were collected by centrifuge, washed three times with ethanol, and dried in a vacuum oven at $80^{\circ} \mathrm{C}$.

\subsection{Compounding of silica nanoparticles with PLA}

A co-rotating miniature twin screw extruder (Haake Mini Lab Rheomex CTW5) was used to disperse the synthesized nanoparticles in PLA matrices at $180^{\circ} \mathrm{C}$ at 
$150 \mathrm{rpm}$. The residence time of the polymer in the compounder was set based on thermal analysis and sample testing to make sure there was no polymer degradation during compounding.

\subsection{Characterization}

\subsubsection{Interfacial tension and contact angle measurements}

Interfacial tension of PLA composites at various pressures of supercritical $\mathrm{CO}_{2}$ and at $153^{\circ} \mathrm{C}(426 \mathrm{~K})$ was measured using axisymmetric drop shape analysis profile (ADSA-P) technique. In brief, the interfacial tension of the polymer samples was measured by fitting the shape and dimensions of their menisci to the theoretical drop profile based on the Laplace equation of capillarity:

$\Delta P=\gamma\left(\frac{1}{R 1}+\frac{1}{R 2}\right)$

where $\gamma$ is interfacial tension, $\Delta P$ is the pressure difference across the interface, and $\mathrm{R} 1$ and $\mathrm{R} 2$ are two principal radii of curvature. If gravity is the only external force, $\Delta P$ can be expressed as a linear function of height:

$$
\Delta P=\Delta P_{0}+(\Delta \rho) g z
$$

where $\Delta P_{0}$ is the pressure difference at a reference plane, and $\mathrm{z}$ is the vertical height of the drop measured from a reference plane. ${ }^{38}$

To do interfacial tension measurements, a high-temperature and high-pressure chamber was designed as explained previously. ${ }^{39-41}$ The accuracy of the interfacial tension measurement was tested using a drop of pure water, and a consistent value of $72.12 \pm 0.11 \mathrm{~mJ} . \mathrm{m}^{-2}$ was evident. For a typical measurement, a small amount of polymer nanocomposite was attached to the tip of a stainless steel rod with a polished tip to avoid asymmetric drop formation. In order to measure the interfacial tension, the density of the drop has to be introduced as an input through the capillary constant: $C=\frac{\Delta \rho g}{\gamma}$, where $\mathrm{C}$ is the capillary constant, $\Delta \rho$ is the density difference between the polymer and the supercritical fluid, $\gamma$ is interfacial tension, and $g$ is acceleration due to gravity. The density of the polymer-gas mixture was calculated using the volume of the drop obtained from the pendant drop profile 
using ADSA software, ${ }^{42}$ and the mass of the drop which is known via the initial weight of the polymer and the amount of absorbed carbon dioxide (at any pressure and temperature) obtained by solubility measurements. The simultaneous measurement of interfacial tension included introduction of the density of the sample after measurement of its volume. Using the new density and through the capillary constant, interfacial tension values can be recalculated. ${ }^{42-44}$ Contact angle measurements were also carried out in a similar high pressure high temperature chamber capable of mounting the silicon surface on top of the inversed stainless steel rod.

\subsubsection{Characterization of the nanoparticles}

Field emission scanning electron microscopy (FE-SEM) (Ultra, Zeiss) with energy-dispersive X-ray (EDX) spectroscopy was used to investigate the morphology of the nanoparticles. The samples were gold-sputtered prior to SEM. SEM images of the solidified PLA-silica APTES nanocomposite melts are also measured through gold-sputtering of the solidified sample after the interfacial tension measurement. A sufficient amount of time is provided to assure the equilibrium interfacial tension is provided.

The hydrodynamic diameter of silica nanoparticles were determined by dynamic light scattering (DLS) on a Zetasizer Nano ZS (Malvern Instruments, Worcestershire, U.K.) equipped with a $4 \mathrm{~mW} \mathrm{He}-\mathrm{Ne}$ laser operating at $633 \mathrm{~nm}$. FTIR spectrums were obtained using a Bruker Vertex 70 FTIR spectrometer from 400 to $4000 \mathrm{~cm}^{-1}$ on a $\mathrm{KBr}$ pellet.

A Dimension Icon ${ }^{\circledR}$ AFM (Bruker Nano Surfaces) with a silicon nitride tip (type SCANASYST-AIR, Bruker) with a radius of $2 \mathrm{~nm}$ was used for AFM imaging in the PeakForce ${ }^{\circledR}$ QNM mode.

\section{Results and Discussions}

The shape and size of the synthesized silica nanoparticles can be observed in SEM and TEM images Figure 1A and B. The uniform spherical particles have an average diameter of around $80 \mathrm{~nm}$ as confirmed with DLS results in Figure $1 \mathrm{C}$ (number-average diameter $\sim 80 \mathrm{~nm}$ and z-average diameter $\sim 100 \mathrm{~nm}$ ). 
FTIR spectra in Figure 2 prove the presence of modification on the silica nanoparticles. In the spectra, the Si-O peaks at 800 and $1100 \mathrm{~cm}^{-1}, \mathrm{Si}-\mathrm{OH}$ at 950 $\mathrm{cm}^{-1}$, and the broad $\mathrm{OH}$ peak in range of 3200 to $3700 \mathrm{~cm}^{-1}$ can be observed in both silica and silica-APTES. However, after surface modification with APTES, the intensity of $\mathrm{OH}$ peak is decreased showing a reduction in the number of surface $\mathrm{OH}$ groups. It is noteworthy to mention that the $\mathrm{OH}$ peak can be attributed to both surface hydroxyl groups and adsorbed water molecules. There are a significant number of hydroxyl groups in terms of isolated silanols, germinals, and vicinals as well as hydrogen bonded hydroxyl groups. The number of hydroxyl groups on the surface varies depending on the hydroxylation state of silica and can be as high as 4.9 OH.nm ${ }^{-1}$ even after vacuum treatment at high temperatures. ${ }^{45}$ On the other hand, there are always water molecules accompanying the $\mathrm{OH}$ groups on the surface as the peak at $1630 \mathrm{~cm}^{-1}$ reveals in the spectrum. These water molecules can only be removed with high temperature thermal treatment. Once they are removed from the surface, the water molecules will immediately adsorb to the surface from the atmosphere and their presence on the surface seems inevitable. ${ }^{46}$ For the silica modified with APTES, bending of $-\mathrm{CH}_{3}$ at $1385 \mathrm{~cm}^{-1}$, stretching of $\mathrm{C}-\mathrm{H}$ at $2933 \mathrm{~cm}^{-1}$ for alkanes, and bending of N-H at $694 \mathrm{~cm}^{-1}$ are recognizable. The Si-OH peak at $950 \mathrm{~cm}^{-1}$ is also decreased significantly. 

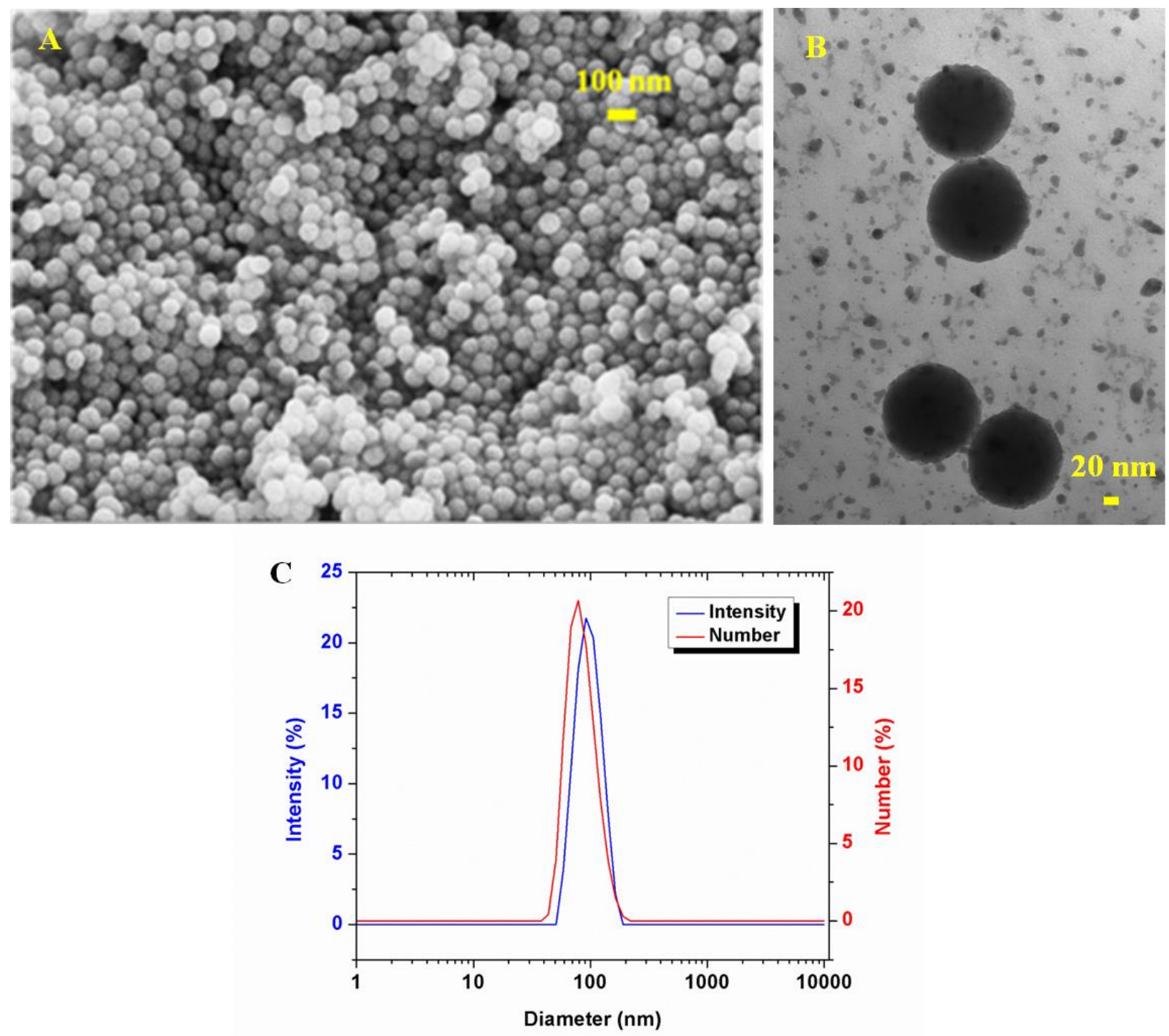

Figure 1.Scanning Electron Microscopy (SEM) (A) and Transmission Electron Microscopy $(T E M)(B)$ images of synthesized silica nanoparticles $(C)$ intensity and number diameter distribution results of the silica nanoparticles obtained from DLS. 

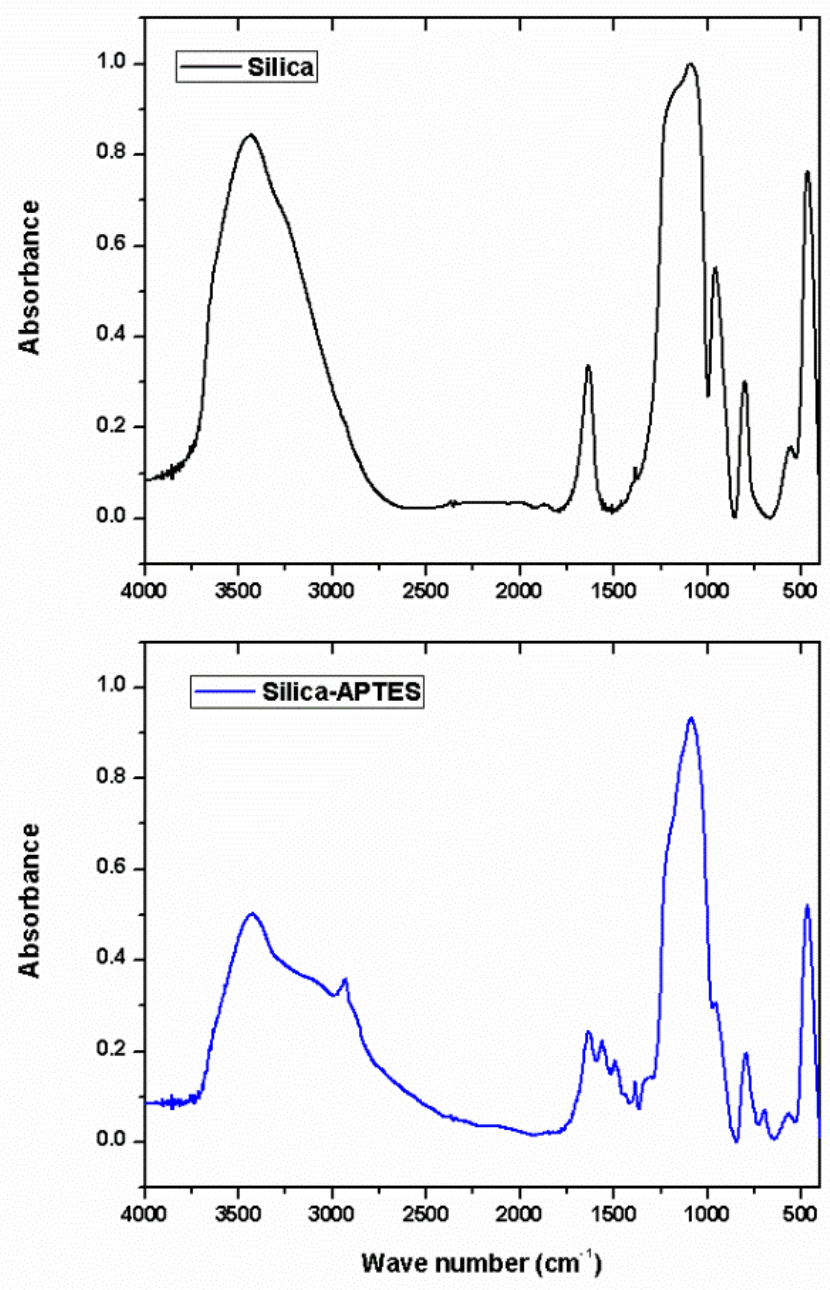

Figure 2. FTIR spectrum of silica nanoparticles before (top) and after (bottom) surface modification with APTES.

In Figure 3, the surface tension values of PLA-silica composites versus silica content at pressures of $\mathrm{CO}_{2}$ ranging from 0 to $13790 \mathrm{kPa}$ (gauge pressure) is illustrated. As can be seen, the interfacial tension decreases with an increase in silica content up to $2 \mathrm{wt}$. \% and then an increase and a plateau is observable in all the pressures. The decrease in interfacial tension can be attributed to the adsorption of the silica nanoparticles to the PLA/ carbon dioxide interface. It has been observed that nanoparticles dispersed in a polymer matrix migrate to a crack generated at the interface between the polymer and a glassy layer. ${ }^{33}$ Thompson et al. ${ }^{34}$ showed that the probability of finding a nano-crystal in the vicinity of the 
polymer surface is higher than the bulk. Nano-crystals form a narrower boundary at an interface with a fluid which lowers the internal energy and thus the interfacial tension. Furthermore, a reduction in surface tension of an air-water interface in a low concentration of nanoparticles as a result of a decrease in internal energy of the interface has been reported. The phenomena has been observed for suspensions of titanium oxides ${ }^{24}$, and silica ${ }^{35}$ at basic $\mathrm{pH}$ values where there is a minimum in interfacial tension versus concentration curve around 5-7 wt.\% of the particles. For both cases, eventually the interfacial tension value will be constant after 10-12 wt. $\%$. Notwithstanding the evidences for adsorption of the nanoparticles to the interface, the reduction in interfacial tension is not as significant as silica in oilwater systems ${ }^{50}$. The reason can be explained in the mechanism by which the nanoparticles act to decrease the interfacial tension; unlike surfactants, particles are driven to the interfaces to remove contact between the two phases ${ }^{51}$ and where the nanoparticles' contact area with the interface is small then the effect on interfacial tension will be less ${ }^{52}$.

As a general fact, nanoparticles can be used as a surface active agent in stabilizing foams and emulsions. ${ }^{8,27,53}$ There are a few reasons for adsorption of nanoparticles to the PLA-CO $\mathrm{CO}_{2}$ interface: first of all, for a particle in a two phase system, due to weaker interactions compared with bulk, creating a surface is more favorable at the interface rather than any of the phases (with the same molecules surrounding). Secondly, the adsorption is thermodynamically favorable not only because of a desirable interaction between amine groups on silica surfaces and carbon dioxide, but also because of an increase in entropy of the polymer bulk as a result of increase in free volume and a reduction in entropy-restricting polymer adsorption at the particle interfaces. ${ }^{54}$

As can be seen in Figure 3 and Figure 4, the interfacial tension increases for values higher than 2 wt. \% of silica. An increase in interfacial tension after a certain loading of nanoparticles can be related to the lateral capillary force created by a deformation of the PLA melt meniscus as a result of partial immersion of silica nanoparticles in the interface. ${ }^{24,35,37,38}$

It is noteworthy that observation of a minimum in interfacial tension-concentration curves should not be mistaken for being the same as the case of aqueous solution of surfactants ${ }^{39}$. In the latter case, the minimum is seen before the critical micelle concentration (CMC) for surface-active impurities in the surfactant system. Due to the presence of nanoparticles at the interface, the shape of interface surrounding them at the fluid phase boundaries is perturbed and is not flat; this fact causes a significant distant dependent capillary force between the particles. ${ }^{25}$ Deformation, 
and subsequent capillary forces are related to the wetting behavior of the particles (contact angle between three phases) and the distance between particles. ${ }^{37}$ The immersion force, the force between small particles partially immersed in both phases, can be attractive or repulsive depending on the sign of the meniscus slope angles at the two contact lines of the two particles adjacent to each other: if the product of the sine of the contact angles for the two particles are positive (negative), the capillary force is going to be attractive (repulsive). ${ }^{37}$ For similar silica nanoparticles with similar surface properties, the contact angles are the same and consequently the force is attractive, provided that the particles are close enough to each other. As can be seen in SEM images in Figure 5, in higher loadings of silica, the number of the nanoparticles is higher at the interface of solidified PLA nanocomposits, and the chance of finding particles close enough to induce an attractive capillary force towards each other is higher. The high capillary force at the interface resists a deformation or stretching in surface area. Since interfacial tension is defined as the work required against a change in the surface area, an increase in the resistance at the interface leads to an increase in the force required to increase the surface (due to capillary force in our case) which eventually causes an increase in interfacial tension in higher loadings of the particles. ${ }^{24}$ 


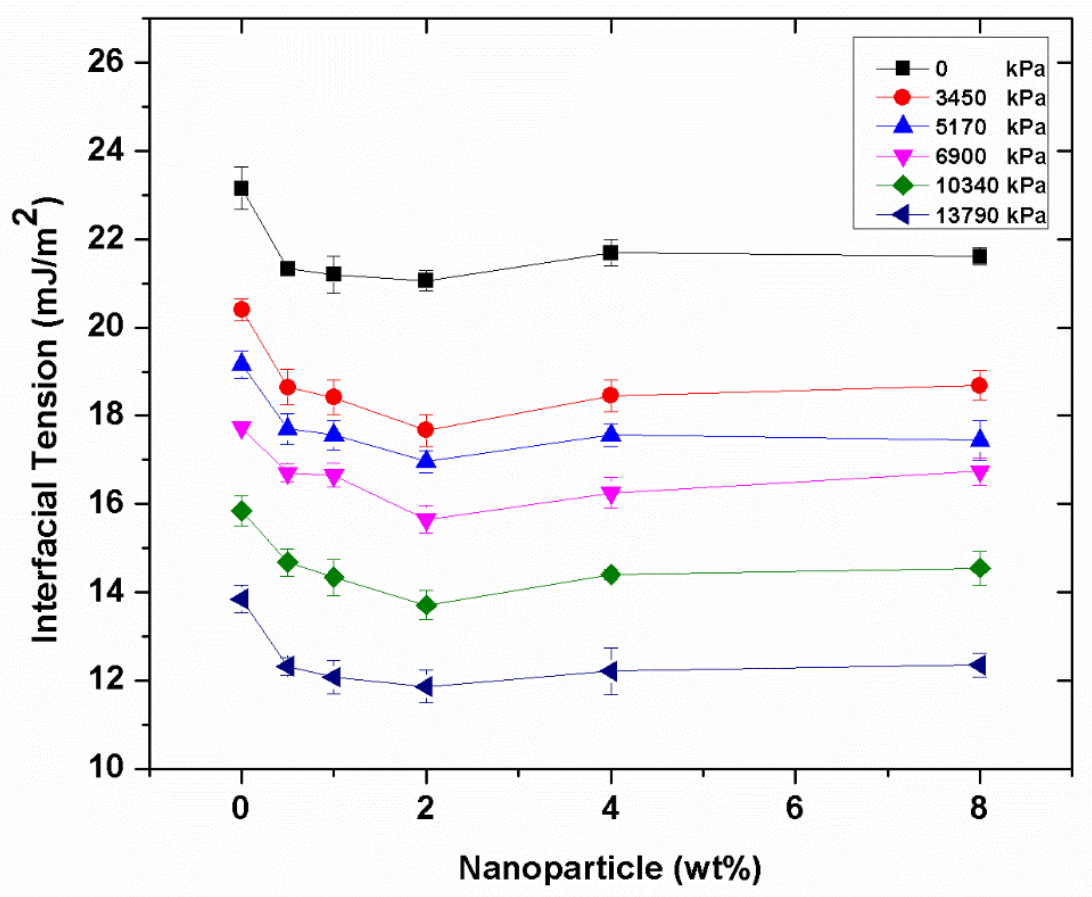

Figure 3. Interfacial tension values of PLA-silica APTES composites versus silica content at different pressures of carbon dioxide (gauge pressure) at $153^{\circ} \mathrm{C}(426 \mathrm{~K})$.

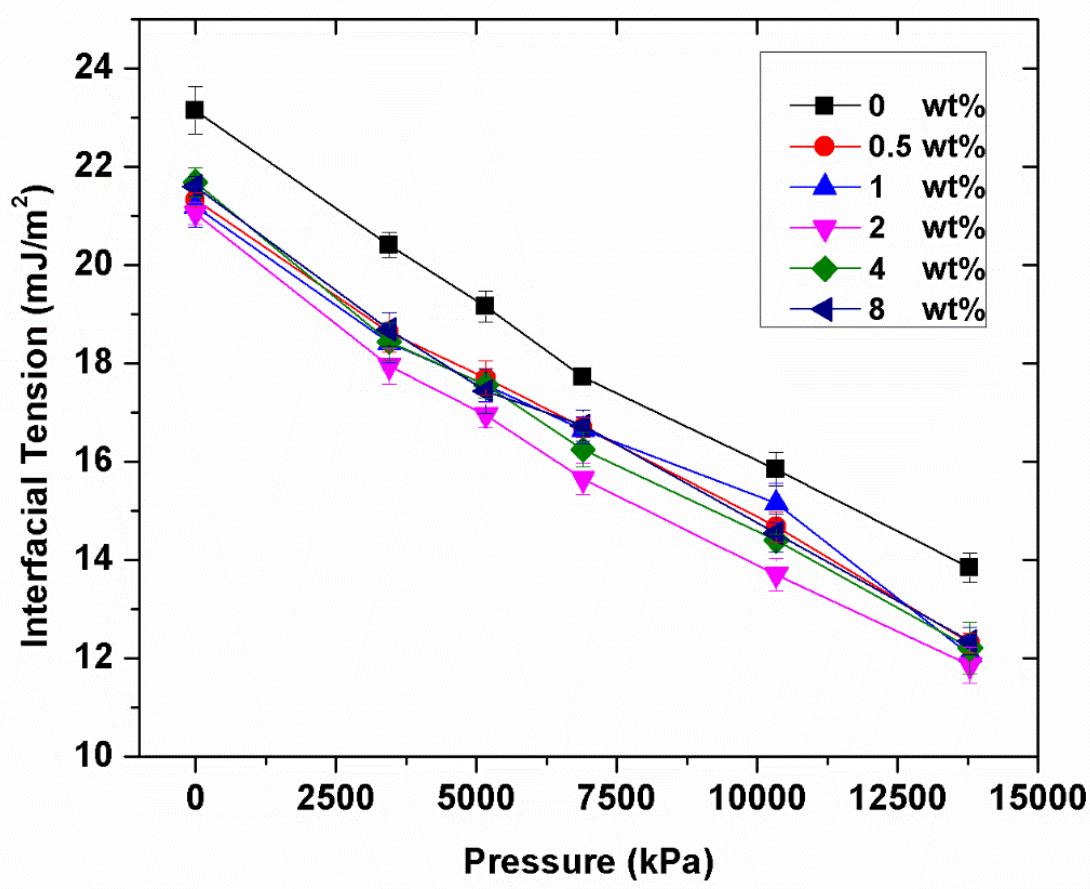

Figure 4. Interfacial tension values of PLA-silica APTES composites versus different pressures of carbon dioxide for various loadings of silica nanoparticles at $153^{\circ} \mathrm{C}(426 \mathrm{~K})$. 
In order to prove the presence of the silica nanoparticles at the interface, SEM images of the solidified nanocomposites were obtained after interfacial tension measurements at atmospheric pressure of carbon dioxide environment and consequently solidification of the pendant drop polymer melt. Figure 5 shows the SEM results for different loadings of APTES-modified silica-PLA nanocomposites. It is clear in SEM images that in 4 and $8 \mathrm{wt} . \%$ of the nanoparticles, the number of the nanoparticles increases significantly which leads to an increase in the chance of finding nanoparticles in vicinity of another particle: particles separated by distances less than the capillary length cause localized lateral capillary forces at higher loadings of the nanoparticles a dominant force to be considered. Considering Equation 3, at higher loadings of the nanoparticles, the two conditions for capillary forces to be effective are available: the distance between the particles in nanoparticle systems are lower than the capillary length $\mathrm{L}$ $<\mathrm{q}^{-1}$ and the radii of the two contact lines is much smaller that the particle separation. It should be noted that even though the surface is more covered at higher loadings of nanoparticles ( $8 \mathrm{wt} . \%)$, the surface coverage is much less than aqueous systems (typically more that $90 \%$ coverage) ${ }^{28}$, therefore the nanoparticle separation can be considered large. EDX results for a surface of $0.5 \mathrm{wt} . \%$ nanocomposite in

Figure 6 shows the presence of a silicon peak for bright spots, proving the spots are silica nanoparticles.
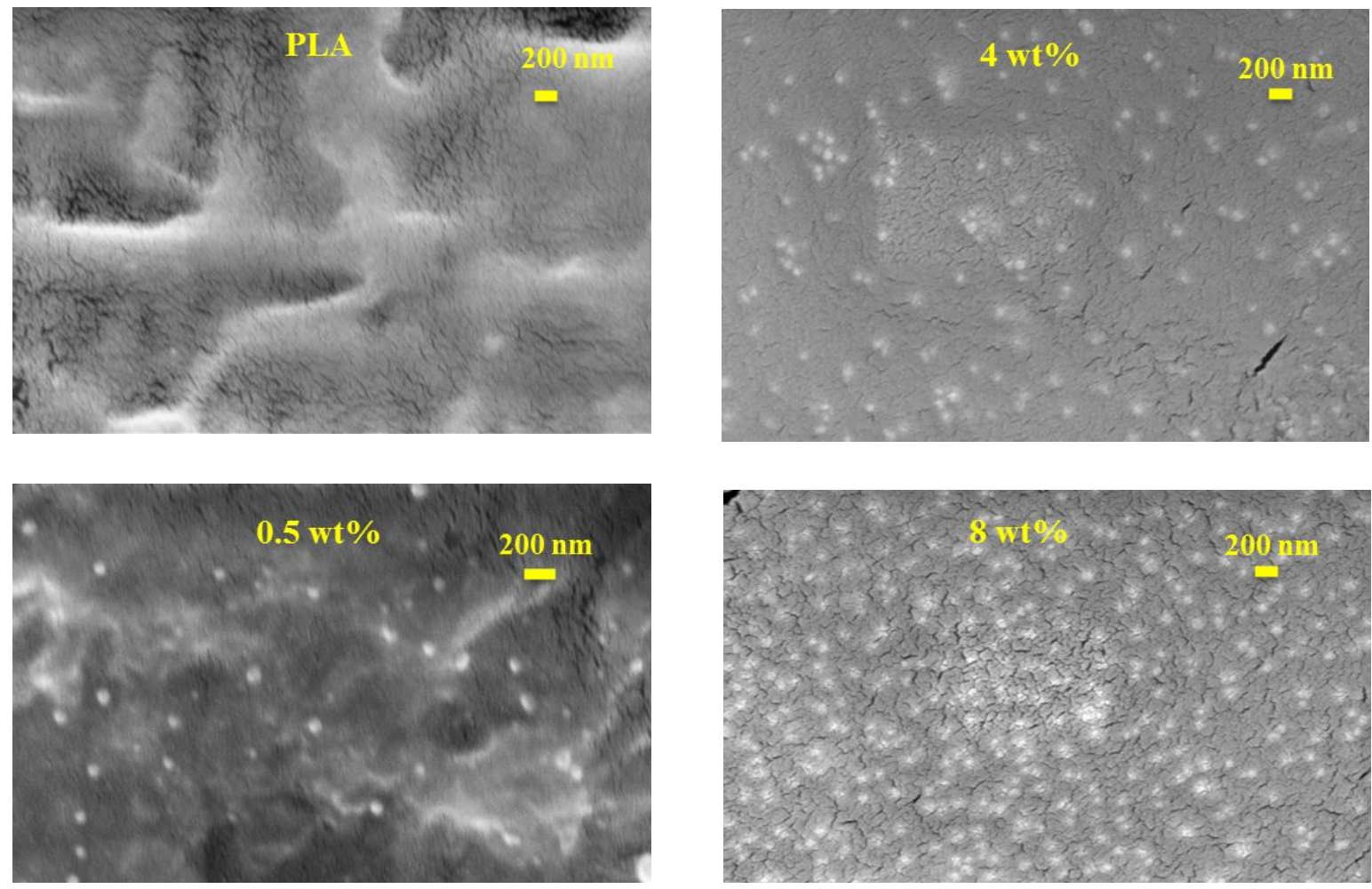

Figure 5. SEM images of solidified PLA-silica APTES nanocomposite melts with different loading of silica after interfacial tension measurement. 

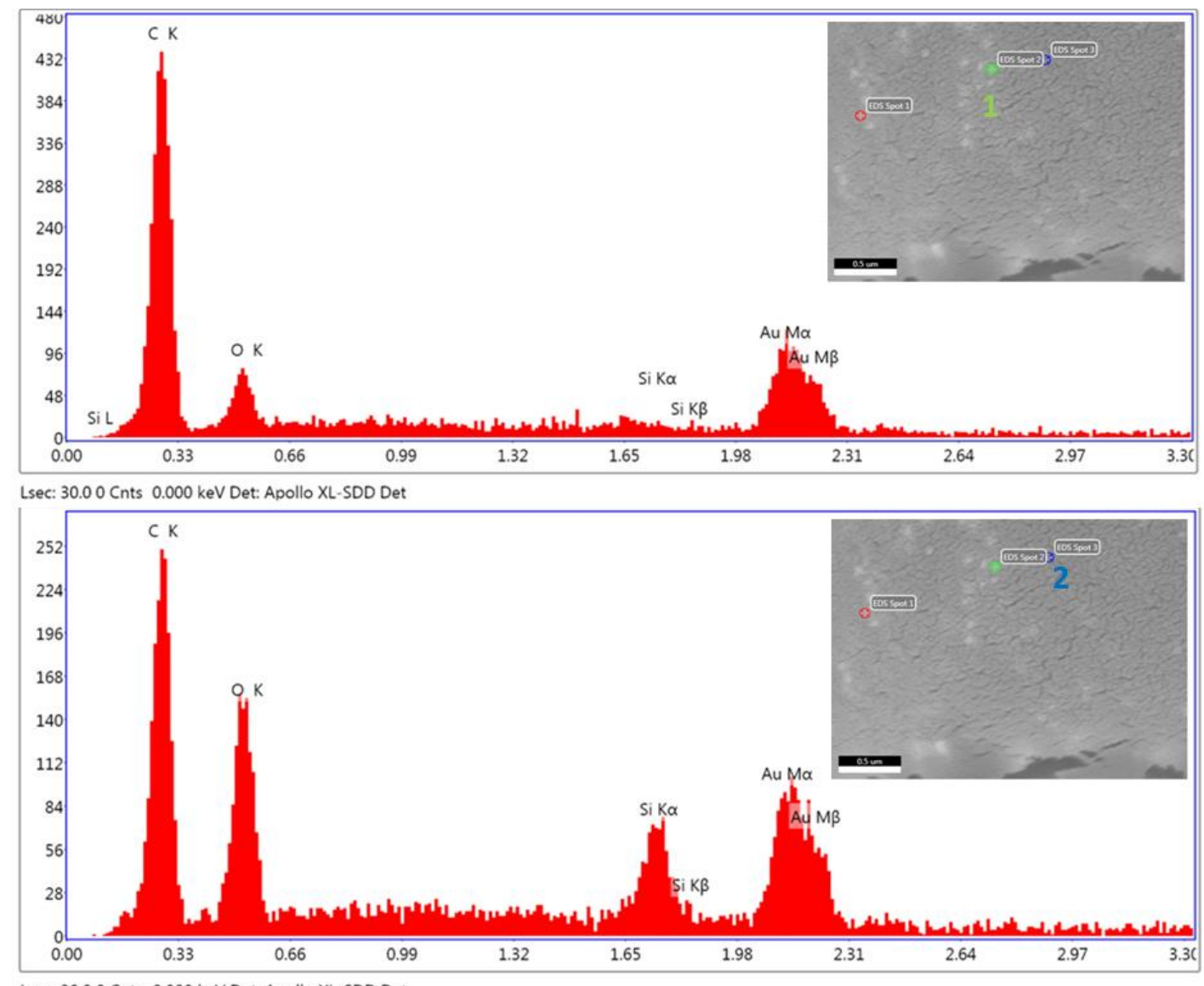

Lsec: 30.00 Cnts 0.000 keV Det: Apollo XL-SDD Det

Figure 6. EDX spectroscopy of spot 1 (top) and spot 2 (bottom) of PLA nanocomposites after being at $153^{\circ} \mathrm{C}(426 \mathrm{~K})$ for the length of the pendant drop measurement.

In addition to direct observation of nanoparticles at the interface, one can show that the adsorption of nanoparticles is thermodynamically favorable and irreversible. It has been shown that if the binding energy of nanoparticles (the energy to detach particles from the interface) is high enough (compared with thermal fluctuations) the adsorption is irreversible. ${ }^{7,9,40}$

Equation 1 is used to calculate binding energy of the particles at the interface. In addition to SEM, this is another proof showing irreversible adsorption of the nanoparticles to a PLA melt-air or supercritical $\mathrm{CO}_{2}$ interface. Knowing the interfacial tension between PLA- supercritical $\mathrm{CO}_{2}$ at the desired temperature and pressure, interfacial tension at $0 \mathrm{wt}$. \% in Figure 3 or Figure 4, the only unknown is the contact angle between the particles at the PLA melt and supercritical $\mathrm{CO}_{2}$ 
interface. In order to measure the contact angle between silica-PLA-CO $\mathrm{CO}_{2}$, one can make a pellet out of the particle and perform the contact angle measurement. However, due to the porosity of the pellet, the liquid or polymer melt on top will diffuse into the pellet making the measurement impossible. To overcome this difficulty, surfaces with the exact surface chemistry of silica and silica modified with APTES can be used for contact angle measurements. Silicon has a natural oxide layer on the surface which after washing with Piranha solution and removing hydrocarbons, has an abundant number of hydroxyl groups on the surface. ${ }^{59,60} \mathrm{In}$ this work, a silicon wafer with surface modification is used as a homologous surface to modified silica.

AFM images of the silicon wafer before and after surface modification with APTES is shown in Figure 7. Both surfaces are uniform and smooth as the root mean square $\left(\mathrm{R}_{\mathrm{q}}\right)$ and arithmetic average $\left(\mathrm{R}_{\mathrm{a}}\right)$ roughness values for silicon wafers are 0.3 and $0.2 \mathrm{~nm}$ and those of silicon modified with APTES are 9.6 and 7.5, respectively. As shown previously, ${ }^{61,62}$ contact angle measurements are independent of surface roughness for values less than $150 \mathrm{~nm}$.
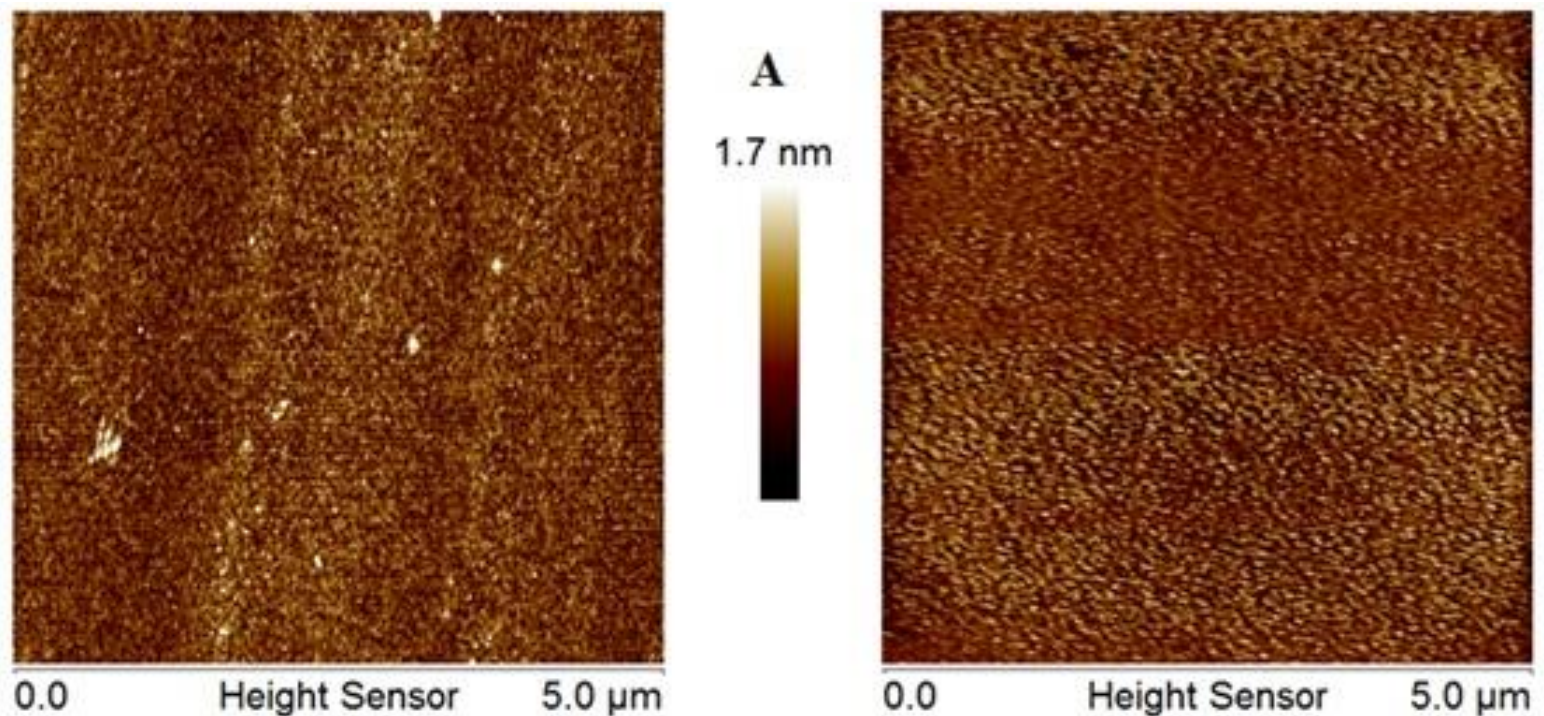

Figure 7. AFM images of silicon (A) and silicon-APTES (B). 


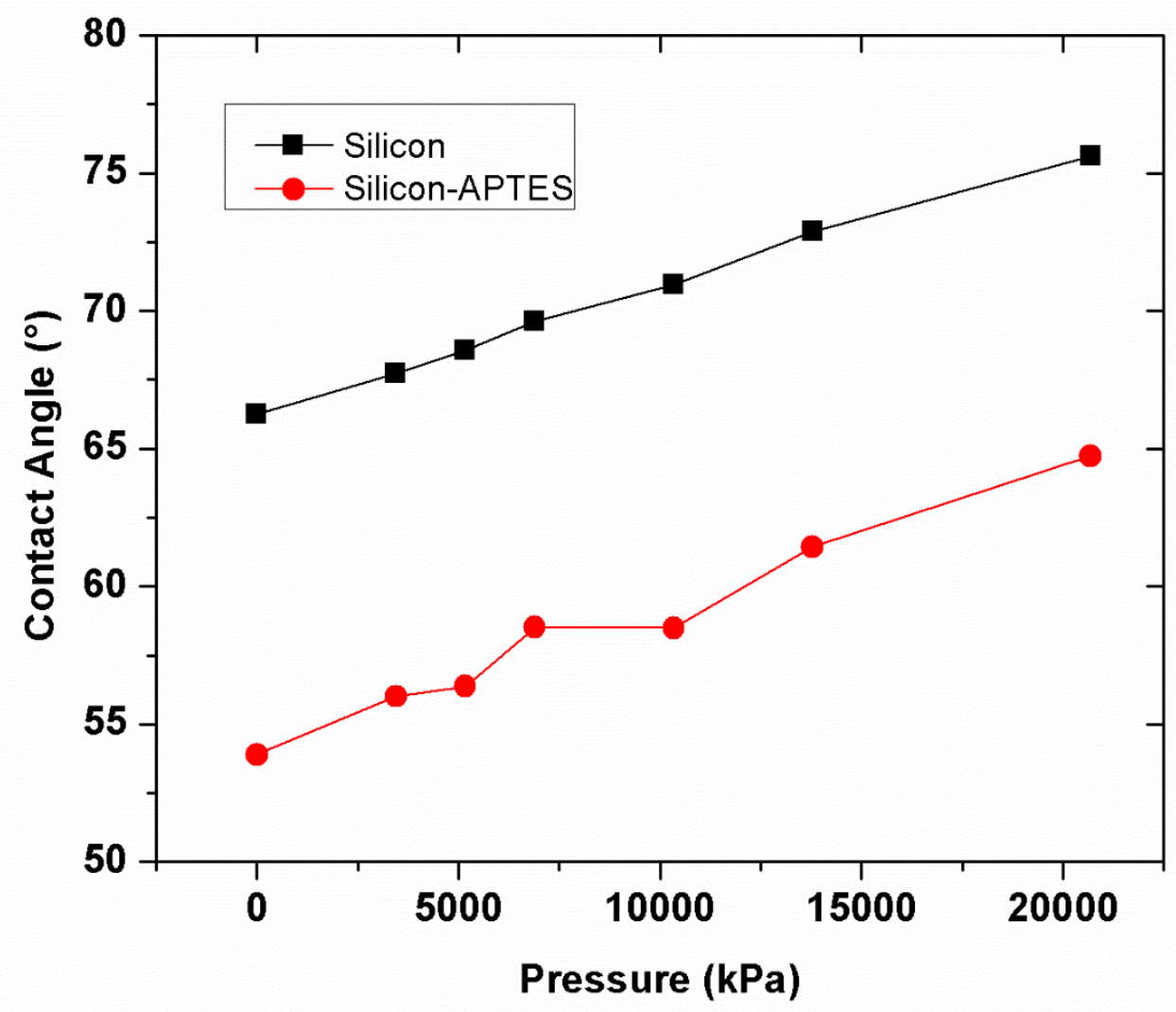

Figure 8. Plot of contact angle of PLA on silicon (black squares) and silicon-APTES (red circles) surfaces vs. pressure of surrounding $\mathrm{CO}_{2}$ at $153^{\circ} \mathrm{C}(426 \mathrm{~K})$ (the maximum value for error bar is $\pm 0.2^{\circ}$ for $95 \%$ confidence interval). 


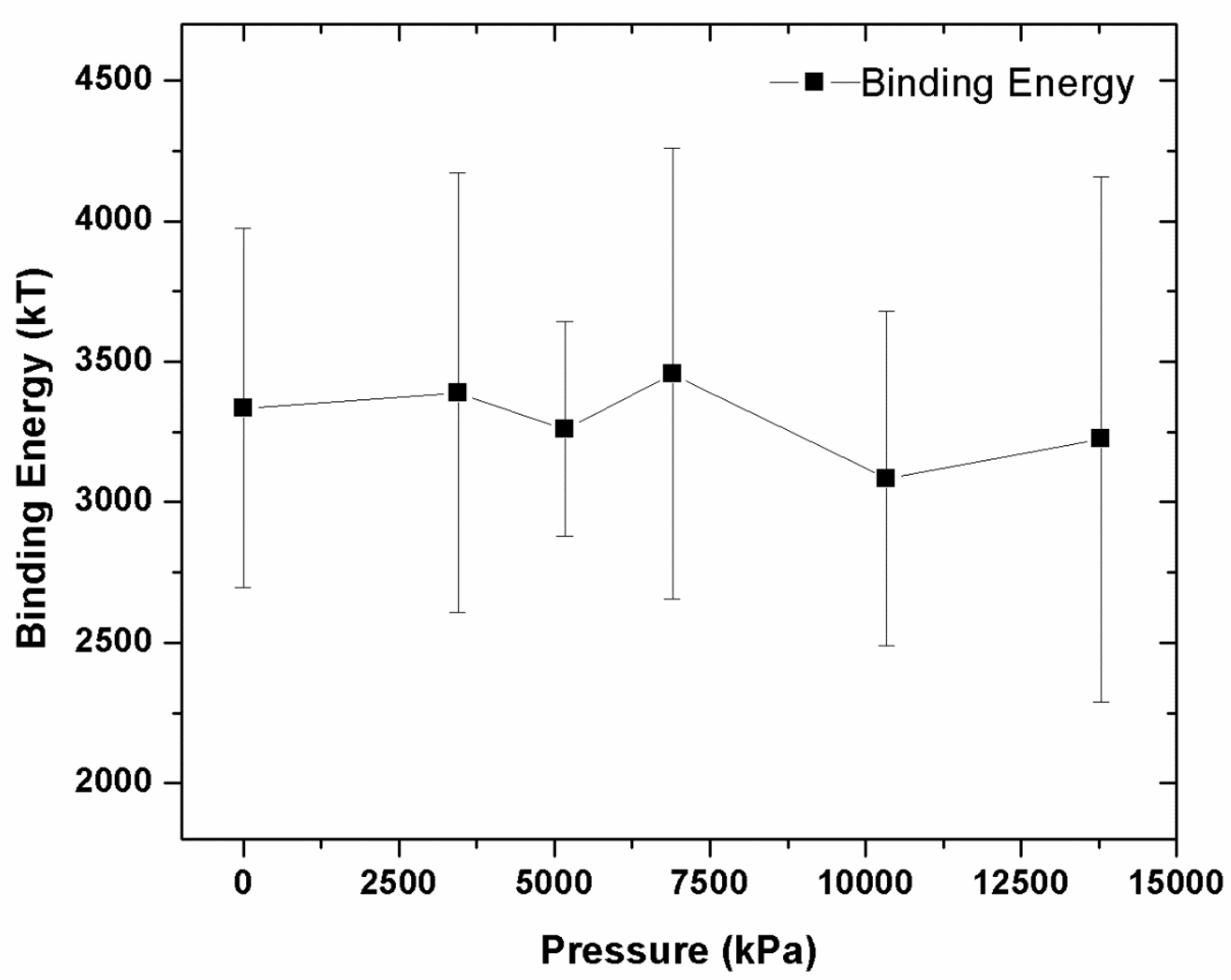

Figure 9.Plot of calculated binding energy of silica-APTES nanoparticles at the PLA- $\mathrm{CO}_{2}$ interface using contact angle results as a function of $\mathrm{CO}_{2}$ pressure at $153^{\circ} \mathrm{C}(426 \mathrm{~K})$.

Figure 8 shows the contact angle of PLA on silica-APTES surfaces as a function of the pressure of surrounding $\mathrm{CO}_{2}$ at $153^{\circ} \mathrm{C}(426 \mathrm{~K})$. The silica-APTES contact angle increases with an increase in $\mathrm{CO}_{2}$ pressure. In other words, the compatibility of nanoparticles with PLA decreases at higher pressures. However, because of a more favorable interaction between the amine group of APTES and PLA, its contact angle is lower. In both cases, the binding energy in Figure 9 is significantly higher than the thermal energy $\left(\mathrm{k}_{\mathrm{B}} \mathrm{T}\right)$, where $\mathrm{k}_{\mathrm{B}}$ is the Boltzmann constant and $\mathrm{T}$ is temperature in Kelvin, making the adsorption irreversible. Based on contact angle and binding energy results, it can be concluded that as the pressure of $\mathrm{CO}_{2}$ increases and the contact angle decreases, the work of adhesion will be less, and consequently there is less tendency towards bulk PLA for nanoparticles, the condition which leads to more adsorption of nanoparticles at the interface. The 
affinity of the nanoparticles towards the interface can be explained in terms of an entropy penalty of polymer chains near the nanoparticles in the presence of $\mathrm{CO}_{2}$; any constraint on polymer configurations such as stretching in the vicinity of a particle, causes a decrease in the conformational entropy of polymer chains, a phenomena that leads to the segregation of the nanoparticles at the polymer-gas interface to minimize the entropic penalty. ${ }^{47,63,64}$ The "entropy-driven" segregation is more pronounced when the polymer chains are expanded as a result of gas absorption specifically near the critical point of the gas. ${ }^{65,66}$

\section{Conclusions}

In summary, the effect of synthesized silica nanoparticles on interfacial tension between PLA and supercritical $\mathrm{CO}_{2}$ at high temperature and high pressures was studied. In the interfacial tension curve, a minimum in silica loading of $2 \mathrm{wt}$. \% for all the pressures was observed, and for higher amounts of silica the interfacial tension value reached a plateau. Adsorption of the silica nanoparticles decreased the interfacial tension, however for higher amounts (more than $2 \mathrm{wt} . \%$ ) of nanoparticles, interfacial tension increased due to attractive lateral capillary forces originating from the perturbation of the PLA- $\mathrm{CO}_{2}$ interface by particles. Contact angle measurements at high temperatures and pressures showed a decrease in compatibility between the nanoparticles and PLA with increasing $\mathrm{CO}_{2}$, which facilitated the adsorption phenomena at high pressures. Furthermore, binding energy calculations showed irreversible adsorption due to high values of energy compared with thermal fluctuations.

\section{Acknowledgment}

The authors cordially acknowledge the financial support from the Natural Sciences and Engineering Research Council (NSERC) through the Network for Innovative Plastic Materials and Manufacturing Processes (NIPMMP) and Canada Research Chairs (CRC).

We thank Chunghwan (Christian) Park and Han Kyul (Sue) Oh for their assistance in processing of the contact angle results. 


\section{References}

(1) Sinha Ray, S.; Okamoto, M. Polymer/layered Silicate Nanocomposites: A Review from Preparation to Processing. Prog. Polym. Sci. (Oxford), 2003, 28, 1539-1641.

(2) Moniruzzaman, M.; Winey, K. I. Polymer Nanocomposites Containing Carbon Nanotubes. Macromolecules, 2006, 39, 5194-5205.

(3) Sarikhani, K.; Abdollahi, S.; Garmabi, H. Preparation of PE Nanocomposites Using Pristine Nano Clay via Alkyl Ammonium Solution Injection in a Twin-Screw Extruder. $J$. Appl. Polym. Sci. 2012, 124, 1344-1351.

(4) Jeddi, K.; Sarikhani, K.; Qazvini, N. T.; Chen, P. Stabilizing Lithium/sulfur Batteries by a Composite Polymer Electrolyte Containing Mesoporous Silica Particles. J. Power Sources 2014, 245, 656-662.

(5) Nasseri, R.; Mohammadi, N. Starch-Based Nanocomposites: A Comparative Performance Study of Cellulose Whiskers and Starch Nanoparticles. Carbohydr. Polym., 2014.

(6) Dinsmore, A. D.; Hsu, M. F.; Nikolaides, M. G.; Marquez, M.; Bausch, A. R.; Weitz, D. A. Colloidosomes: Selectively Permeable Capsules Composed of Colloidal Particles. Science 2002, 298, 1006-1009.

(7) Niu, Z.; He, J.; Russell, T. P.; Wang, Q. Synthesis of Nano/microstructures at Fluid Interfaces. Angew. Chem. Int. Ed, 2010, 49, 10052-10066.

(8) Dickinson, E. Food Emulsions and Foams: Stabilization by Particles. Curr. Opin. Colloid Interface Sci., 2010, 15, 40-49.

(9) Aveyard, R.; Binks, B. P.; Clint, J. H. Emulsions Stabilised Solely by Colloidal Particles. Adv. Colloid Interface Sci. 2003, 100-102, 503-546.

(10) Gonzenbach, U. T.; Studart, A. R.; Tervoort, E.; Gauckler, L. J. Stabilization of Foams with Inorganic Colloidal Particles. Langmuir 2006, 22, 10983-10988.

(11) Eaves, D. Handbook of Polymer Foams; Eaves, D., Ed.; Rapra Technology Limited, 2004.

(12) Saeidlou, S.; Huneault, M. a.; Li, H.; Park, C. B. Poly(lactic Acid) Crystallization. Prog. Polym. Sci. 2012, 37, 1657-1677.

(13) Auras, R.; Harte, B.; Selke, S. An Overview of Polylactides as Packaging Materials. Macromol. Biosci. 2004, 4, 835-864.

(14) Dorgan, J. R.; Lehermeier, H. J.; Palade, L.-I.; Cicero, J. Polylactides/PP : Properties and Prospects of an Environmentally Benign Plastic from Renewable Resources. Macromol. Symp. 2001, 175, 55-66. 
(15) Li, H.; Lee, L. J.; Tomasko, D. L. Effect of Carbon Dioxide on the Interfacial Tension of Polymer Melts. Ind. Eng. Chem. Res. 2004, 43, 509-514.

(16) Liao, X.; Li, Y. G.; Park, C. B.; Chen, P. Interfacial Tension of Linear and Branched PP in Supercritical Carbon Dioxide. J. Supercrit. Fluids 2010, 55, 386-394.

(17) Lee S, Park C. B., Ramesh N. S. Polymeric Foams: Science and Technology; Taylor and Francis Group, LLC, 2007.

(18) Kim, Y.; Park, C. B.; Chen, P.; Thompson, R. B. Maximal Cell Density Predictions for Compressible Polymer Foams. Polymer. 2013, 54, 841-845.

(19) Kim, Y.; Park, C. B.; Chen, P.; Thompson, R. B. Towards Maximal Cell Density Predictions for Polymeric Foams. Polymer. 2011, 52, 5622-5629.

(20) Wong, A.; Wijnands, S. F. L.; Kuboki, T.; Park, C. B. Mechanisms of Nanoclay-Enhanced Plastic Foaming Processes: Effects of Nanoclay Intercalation and Exfoliation. $J$. Nanoparticle Res. 2013, 15.

(21) Leung, S. N.; Wong, A.; Wang, L. C.; Park, C. B. Mechanism of Extensional StressInduced Cell Formation in Polymeric Foaming Processes with the Presence of Nucleating Agents. J. Supercrit. Fluids 2012, 63, 187-198.

(22) Nofar, M.; Tabatabaei, A.; Park, C. B. Effects of Nano-/micro-Sized Additives on the Crystallization Behaviors of PLA and PLA/CO2 Mixtures. Polymer (United Kingdom) 2013, 54, 2382-2391.

(23) Wang, J.; Zhu, W.; Zhang, H.; Park, C. B. Continuous Processing of Low-Density, Microcellular Poly(lactic Acid) Foams with Controlled Cell Morphology and Crystallinity. Chem. Eng. Sci. 2012, 75, 390-399.

(24) Wong, A.; Guo, Y.; Parka, C. B. Fundamental Mechanisms of Cell Nucleation in Polypropylene Foaming with Supercritical Carbon Dioxide - Effects of Extensional Stresses and Crystals. J. Supercrit. Fluids 2013, 79, 142-151.

(25) Binks, B. P.; Murakami, R. Phase Inversion of Particle-Stabilized Materials from Foams to Dry Water. Nat. Mater. 2006, 5, 865-869.

(26) Johnston, K. P.; Rocha, S. R. P. Da. Colloids in Supercritical Fluids over the Last 20 Years and Future Directions. J. Supercrit. Fluids 2009, 47, 523-530.

(27) Binks, B. P. Particles as Surfactants - Similarities and Differences. Curr. Opin. Colloid Interface Sci., 2002, 7, 21-41.

(28) Du, K.; Glogowski, E.; Emrick, T.; Russell, T. P.; Dinsmore, A. D. Adsorption Energy of Nano- and Microparticles at Liquid-Liquid Interfaces. Langmuir 2010, 26, 12518-12522. 
(29) Maestro, A.; Guzmán, E.; Ortega, F.; Rubio, R. G. Contact Angle of Micro- and Nanoparticles at Fluid Interfaces. Curr. Opin. Colloid Interface Sci. 2014, 19, 355-367.

(30) Danov, K. D.; Kralchevsky, P. A. Capillary Forces between Particles at a Liquid Interface: General Theoretical Approach and Interactions between Capillary Multipoles. $A d v$. Colloid Interface Sci., 2010, 154, 91-103.

(31) Casson, K.; Johnson, D. Surface-Tension-Driven Flow Due to the Adsorption and Desorption of Colloidal Particles. J. Colloid Interface Sci. 2001, 242, 279.

(32) Dong, L.; Johnson, D. Surface Tension of Charge-Stabilized Colloidal Suspensions at the Water-Air Interface. Langmuir 2003, 19, 10205-10209.

(33) Kralchevsky, P. A.; Nagayama, K. Capillary Interactions between Particles Bound to Interfaces, Liquid Films and Biomembranes. Adv. Colloid Interface Sci. 2000, 85, 145192.

(34) Bresme, F.; Oettel, M. Nanoparticles at Fluid Interfaces. J. Phys. Condens. Matter 2007, $19,413101$.

(35) Stober, W. Controlled Growth of Monodisperse Silica Spheres in the Micron Size range. J. Colloid Interface Sci. 1968, 26, 62-69.

(36) Nyström, D.; Antoni, P.; Malmström, E.; Johansson, M.; Whittaker, M.; Hult, A. HighlyOrdered Hybrid Organic-Inorganic Isoporous Membranes from Polymer Modified Nanoparticles. Macromol. Rapid Commun. 2005, 26, 524-528.

(37) Vandenberg, E. T.; Bertilsson, L.; Liedberg, B.; Uvdal, K.; Erlandsson, R.; Elwing, H.; Lundström, I. Structure of 3-Aminopropyl Triethoxy Silane on Silicon Oxide. J. Colloid Interface Sci., 1991, 147, 103-118.

(38) Neumann, A. W., David, R., Zuo, Y. (Eds. ). Applied Surface Thermodynamics (Vol. 151).; CRC Press: Taylor \& Francis Group, 2010.

(39) Park, H.; Park, C. B.; Tzoganakis, C.; Tan, K. H.; Chen, P. Surface Tension Measurement of Polystyrene Melts in Supercritical Carbon Dioxide. Ind. Eng. Chem. Res. 2006, 45, $1650-1658$.

(40) Wei, H.; Thompson, R. B.; Park, C. B.; Chen, P. Surface Tension of High Density Polyethylene (HDPE) in Supercritical Nitrogen: Effect of Polymer Crystallization. Colloids Surfaces A Physicochem. Eng. Asp. 2010, 354, 347-352.

(41) Sarikhani, K.; Jeddi, K.; Thompson, R. B.; Park, C. B.; Chen, P. Effect of Pressure and Temperature on Interfacial Tension of Poly Lactic Acid Melt in Supercritical Carbon Dioxide. Thermochim. Acta 2015, 609, 1-6. 
(42) Park, H.; Park, C. B.; Tzoganakis, C.; Tan, K.-H.; Chen, P. Simultaneous Determination of the Surface Tension and Density of Polystyrene in Supercritical Nitrogen. Ind. Eng.

Chem. Res. 2008, 47, 4369-4373.

(43) Sage, D.; Müller, M.; Stalder, A. F.; Melchior, T.; Blu, T.; Unser, M. Low-Bond Axisymmetric Drop Shape Analysis for Surface Tension and Contact Angle Measurements of Sessile Drops. Colloids Surf., A, 2010, 364, 72-81.

(44) Wulf, M.; Michel, S.; Grundke, K.; del Rio OI; Kwok, D.; Neumann, A. Simultaneous Determination of Surface Tension and Density of Polymer Melts Using Axisymmetric Drop Shape Analysis. J. Colloid Interface Sci. 1999, 210, 172-181.

(45) Zhuravlev, L. T. Concentration of Hydroxyl Groups on the Surface of Amorphous Silicas. Langmuir 1987, 3, 316-318.

(46) Hair, M. L. Hydroxyl Groups on Silica Surface. J. Non-Cryst. Solids, 1975, 19, 299-309.

(47) Gupta, S.; Zhang, Q.; Emrick, T.; Balazs, A. C.; Russell, T. P. Entropy-Driven Segregation of Nanoparticles to Cracks in Multilayered Composite Polymer Structures. Nat. Mater. 2006, 5, 229-233.

(48) Thompson, R. B.; Park, C. B.; Chen, P. Reduction of Polymer Surface Tension by Crystallized Polymer Nanoparticles. J. Chem. Phys. 2010, 133, 144913.

(49) Dong, L.; Johnson, D. T. The Study of the Surface Tension of Charge- Stabilized Colloidal Dispersions. J. Dispers. Sci. Technol. 2005, 25, 575-583.

(50) Whitby, C. P.; Fornasiero, D.; Ralston, J.; Liggieri, L.; Ravera, F. Properties of Fatty Amine-Silica Nanoparticle Interfacial Layers at the Hexane-Water Interface. J. Phys. Chem. C 2012, 116, 3050-3058.

(51) Ranatunga, R. J. K. U.; Nguyen, C. T.; Wilson, B. A.; Shinoda, W.; Nielsen, S. O. Molecular Dynamics Study of Nanoparticles and Non-Ionic Surfactant at an Oil-water Interface. Soft Matter, 2011, 7, 6942.

(52) Luo, M.; Dai, L. L. Molecular Dynamics Simulations of Surfactant and Nanoparticle SelfAssembly at Liquid-liquid Interfaces. J. Phys. Condens. Matter, 2007, 19, 375109.

(53) Gonzenbach, U. T.; Studart, A. R.; Tervoort, E.; Gauckler, L. J. Ultrastable ParticleStabilized Foams. Angew. Chemie - Int. Ed. 2006, 45, 3526-3530.

(54) Thompson, R. B.; Ginzburg, V. V; Matsen, M. W.; Balazs, A. C. Predicting the Mesophases of Copolymer-Nanoparticle Composites. Science 2001, 292, 2469-2472.

(55) Kralchevsky, P. a; Nagayama, K. Capillary Forces between Colloidal Particles. Langmuir 1994, 10, 23-36. 
(56) Dong, L.; Johnson, D. Interfacial Tension Measurements of Colloidal Suspensions: An Explanation of Colloidal Particle-Driven Interfacial Flows. Adv. Sp. Res. 2003, 32, 149153.

(57) Lin, S.-Y.; Lin, Y.-Y.; Chen, E.-M.; Hsu, C.-T.; Kwan, C.-C. A Study of the Equilibrium Surface Tension and the Critical Micelle Concentration of Mixed Surfactant Solutions. Langmuir 1999, 15, 4370-4376.

(58) Garbin, V.; Crocker, J. C.; Stebe, K. J. Nanoparticles at Fluid Interfaces: Exploiting Capping Ligands to Control Adsorption, Stability and Dynamics. J Colloid Interf Sci. 2012, 387, 1 .

(59) Turan, E.; Demirci, S.; Caykara, T. Synthesis of Thermoresponsive poly(NIsopropylacrylamide) Brush on Silicon Wafer Surface via Atom Transfer Radical Polymerization. Thin Solid Films 2010, 518, 5950-5954.

(60) Matyjaszewski, K.; Dong, H.; Jakubowski, W.; Pietrasik, J.; Kusumo, A. Grafting from Surfaces for "Everyone": ARGET ATRP in the Presence of Air. Langmuir 2007, 23, 4528-4531.

(61) Khoshkava, V.; Kamal, M. R. Effect of Surface Energy on Dispersion and Mechanical Properties of Polymer/nanocrystalline Cellulose Nanocomposites. Biomacromolecules 2013, 14, 3155-3163.

(62) Hideo Nakae; Ryuichi Inui; Yosuke Hirata; Hiroyuki Saito. Effects of Surface Roughness on Wettability. Acta Materialia, 1998, 46, 2313-2318.

(63) Lee, J.-Y.; Zhang, Q.; Emrick, T.; Crosby, A. J. Nanoparticle Alignment and Repulsion during Failure of Glassy Polymer Nanocomposites. Macromolecules 2006, 39, 73927396.

(64) Tyagi, S.; Lee, J. Y.; Buxton, G. A.; Balazs, A. C. Using Nanocomposite Coatings to Heal Surface Defects. Macromolecules 2004, 37, 9160-9168.

(65) Asada, M.; Gin, P.; Endoh, M. K.; Satija, S. K.; Taniguchi, T.; Koga, T. Directed SelfAssembly of Nanoparticles at the Polymer Surface by Highly Compressible Supercritical Carbon Dioxide. Soft Matter 2011, 7, 9231.

(66) Koga, T.; Gin, P.; Yamaguchi, H.; Endoh, M. K.; Asada, M.; Sendogdular, L.; Kobayashi, M.; Takahara, A.; Akgun, B.; Satija, S. K.; et al. Generality of Anomalous Expansion of Polymer Chains in Supercritical Fluids. Polymer (United Kingdom) 2011, 52, 4331-4336. 